\title{
Stigma, Body Image, and Quality of Life in Women Seeking Orthognathic Surgery
}

\author{
Li-Wei Lee, M.S. \\ Sue-Huei Chen, Ph.D. \\ Chung-Chih Yu, M.D. \\ Lun-Jou Lo, M.D. \\ Sheau-Rong Lee, M.S. \\ Yu-Ray Chen, M.D. \\ Taipei and Taoyuan, Taiwan
}

Background: Orthognathic surgery has been increasingly performed in the authors' country. To ascertain the increased satisfaction that comes with surgery, it is necessary to evaluate the patient's motivation for surgery.

Methods: Seventy-four female patients were evaluated before surgery. The control group consisted of 124 female patients. All participants completed the measures of perception of appearance, body image, stigma, and quality of life on an individual basis. These measures were tested, with satisfactory reliability and validity.

Results: Patients scored significantly lower than the control group on the measures for perception of appearance, stigma of surgery, and quality of life, but higher on appearance orientation and stigma of deformity. Correlation analyses revealed various patterns of intercorrelations of subscales of stigma and body image in both groups. Regression analysis yielded three significant predictors for surgery motivation (i.e., satisfaction of facial areas, stigma of surgery, and appearance orientation), altogether accounting for 46.2 percent of the total variance.

Conclusions: Given the broadly known importance of body image in seeking surgery, it may be equally important to take stigma into consideration of the patient's motivation. Further investigations are needed. (Plast. Reconstr. Surg. 120: 225, 2007.)

\section{-}

he appearance of one's dentition, although only part of the face, can greatly influence facial features and the perception of attractiveness. Dentofacial deformities, such as open bite, overbite, class III malocclusion, deep bite, and crowding are common targets for teasing during childhood.1,2 With the advent of combined orthodontic and surgical treatments, more harmonious facial-skeletal relationships and improved occlusal function can be achieved. It is now more popular for patients with dentofacial deformities to seek orthognathic surgery for aesthetic concerns rather than simply for functional improvement. Existing evidence also suggests that patients undergoing orthognathic surgery may benefit from less social embarrassment, better body image, and improved self-esteem. ${ }^{3-5}$

\footnotetext{
From the Departments of Psychology and Psychiatry, National Taiwan University; Center of GeneralEducation, Chang Gung University; and Craniofacial Center and Department of Child Psychiatry, Chang Gung Memorial Hospital.

Received for publication September 20, 2005; accepted January 12, 2006.

Copyright (2007 by the American Society of Plastic Surgeons

DOI: 10.1097/01.prs.0000264100.57630.c7
}

Between 2001 and 2004, orthognathic surgery has been increasingly performed at our medical center (Table 1). The majority of patients are between 16 and 25 years of age (60 to 70 percent), with a lesser number in the 26- to 35-year age group (20 to 30 percent). Female patients are twice as common as male patients. To ascertain the increased satisfaction that comes with surgery, it is necessary to evaluate the patients' psychosocial status before surgery. Because there is little written information regarding the psychosocial aspects of patients undergoing orthognathic surgery in our country, the purpose of this study was to examine the relationship between body image and surgery in our subjects, including the cultural aspects related to the stigma of surgery, to better understand the motivations for surgery.

Body image has historically focused on the eating disorders and obesity that affect women, but has been broadened recently to include the quality of life for a wide range of physical conditions across a spectrum of medical and health disciplines. ${ }^{6}$ Body image is thought to be multidimensional; however, investment in, and evaluation of, body image are central factors. Body 
Table 1. Orthognathic Surgery Performed from 2001 to 2004 at a Medical Center*

\begin{tabular}{|c|c|c|c|c|c|}
\hline & $2001(\%)$ & $2002(\%)$ & $2003(\%)$ & $2004(\%)$ & Study Group (\%) \\
\hline Totals & 126 & 194 & 227 & 244 & 106 \\
\hline \multicolumn{6}{|l|}{ Age } \\
\hline $16-25 \mathrm{yr}$ & $93(73.8)$ & $122(62.9)$ & $149(65.6)$ & $154(63.1)$ & $62(58.5)$ \\
\hline $26-35 \mathrm{yr}$ & $30(23.8)$ & $58(29.9)$ & $66(29.1)$ & $80(32.8)$ & $38(35.8)$ \\
\hline$\geq 36$ yr & $3(2.4)$ & $10(5.2)$ & $12(5.3)$ & $10(3.1)$ & $6(4.7)$ \\
\hline \multicolumn{6}{|l|}{ Sex } \\
\hline Male & $42(33.3)$ & $54(27.8)$ & $80(35.2)$ & $82(33.7)$ & $28(26.4)$ \\
\hline Female & $84(66.7)$ & $140(72.2)$ & $147(64.8)$ & $162(66.3)$ & $78(73.6)$ \\
\hline
\end{tabular}

*The study was approved by the National Science Council prior to data collection. Between July of 2004 and February of 2005,106 patients who sought orthognathic surgery were interviewed at the medical center. The statistics of this medical center are representative, because the majority of orthognathic surgery was performed at the medical center in our country.

image investment is defined as a measure of the importance of body image as it impacts on one's self-esteem. Body image evaluation is characterized by the degree to which one is satisfied or dissatisfied with one's appearance. ${ }^{7}$

Sarwer et al. have proposed a theoretical model that explains the relationship between body image and the decision to seek cosmetic procedures. The interaction between body image investment and evaluation is thought to influence a person's decision to seek cosmetic treatments. ${ }^{8,9}$ With respect to the attitudes of those who have undergone cosmetic surgery, some studies have found that patients report a higher dissatisfaction with body image and levels of investment similar to a control group before surgery, ${ }^{10}$ whereas other results indicated that patients who had cosmetic surgery had a greater body image investment but a similar level of satisfaction as the control group. ${ }^{11}$ Greater body image investment has been associated with more favorable attitudes toward cosmetic surgery. 8,9

It is possible that the extent to which the two components of body image serve as a patient's motivation for aesthetic surgery depends on the type of deformity. With this in mind, we hypothesized that the study group had a higher investment in seeking orthognathic surgery, but not necessarily greater body dissatisfaction. Dentofacial problems do not represent a deficit involving the whole body; rather, the dissatisfaction may be limited to a specific part. The perception of appearance, a subjective rating of facial appearance, was measured in this study and was worse in the study group.

In earlier days in our country, as in many relatively socially oriented societies, seeking cosmetic improvement was often viewed as a pursuit that brought embarrassment to the individual and his or her family. Consequently, those who sought cosmetic surgery would conceal their intentions. In step with global trends, it has become more popular and acceptable to undergo orthognathic surgery to correct dentofacial deviations and to enhance beauty in recent years.

The stigma of visible deformities, rooted in one's sociocultural background, adversely influences the development of body image and, as a result, increases feelings of social inadequacy. ${ }^{12}$ Rather than a pursuit for physical perfection, some patients seek surgical solutions to elevate the distressed mood that results from repeated experiences that highlight their appearance. ${ }^{13} \mathrm{In}$ other words, the acceptance of cosmetic surgery may be more a function of one's fear of becoming unattractive rather than the hope of becoming more attractive. ${ }^{14}$ We have hypothesized that those interested in orthognathic surgery suffer a higher stigma from their deformities before surgery.

From a pathologic point of view, it is prudent to question whether persons with dentofacial deformity are psychologically unhealthy. Dentofacial deviations have been reported to be associated with an increased risk for psychological maladjustment. ${ }^{4}$ However, some recent research has shown that patients are generally able to adjust to such deformities and report relatively few psychosocial issues before surgery. ${ }^{15}$ Furthermore, the extent of the stigma and psychological maladjustment reported in earlier studies may reflect the popular notion at a time and within a cultural context when dentofacial deviations and cosmetic surgery were less accepted. ${ }^{4}$

Pruzinsky and Cash ${ }^{16}$ discussed the benefits of improving body image as it relates to healthrelated quality of life. This prompted us to ascertain what positive quality-of-life characteristics were difficult for those with dentofacial deformities to achieve. Such an assessment would be of clinical value and assist physicians and members of the treatment team in understanding concerns regarding body image. The goals of the current study, therefore, were to determine (1) the difference between orthognathic surgery pa- 
tients and a control group with respect to perception of appearance, body image, stigma, and quality of life; (2) the correlations among perception of appearance, body image, stigma, and quality of life; and (3) the predictors of motivation for surgery in patients with dentofacial abnormalities.

\section{PATIENTS AND METHODS}

\section{Participants}

Between July of 2004 and February of 2005, 106 patients who sought orthognathic surgery were interviewed at the craniofacial center of a medical center. Because the majority of orthognathic surgery patients were characterized as female and younger than 35 years (Table 1), 74 female patients were enrolled in this study. Their mean age was $24.7 \pm 4.2$ years (range, 17.8 to 34.5 years). In the study group, 92.3 percent were unmarried and 87.8 percent were college educated. The type of dentofacial deformities included 16.2 percent maxillary protrusion with a recessed chin, 20.3 percent bimaxillary protrusions, 54.1 percent mandibular prognathism, and 4.1 percent all other deformities.

To closely match the orthognathic surgery patients, the control group consisted of 124 female college students recruited from two universities. Their mean age was $20.3 \pm 1.5$ years (range, 17.5 to 24.5 years).

\section{Measures for Study and Control Groups \\ Perception of Appearance}

The perception of appearance is a measure that consists of two ratings. The Overall Appearance Rating is a self-rating of one's own overall appearance, ranging from 0 to 100 , with 0,50 , and 100 being extremely ugly, ordinary, and extremely beautiful, respectively. The Satisfaction of Facial Areas asks each participant to rate the extent of his or her satisfaction about six facial parts using a five-point Likert scale, with 1 being very dissatisfied and 5 being very satisfied. The six facial parts included the face, nose, cheek, lip, teeth, and chin, which are most relevant to dentofacial deformities.

\section{Body Image}

The Multidimensional Body-Self Relations Questionnaire is one of the most frequently used body image measures used for the evaluation of one's body image. ${ }^{17,18}$ Two subscales were administrated: (1) the Appearance Evaluation subscale, which measures the evaluative-affective satisfaction toward one's own appearance, with higher scores indicating a greater satisfaction with appearance; and (2) the Appearance Orientation, which assesses the cognitive-behavioral investment in one's own appearance and reflects whether the appearance is psychologically important to the individual and whether her or his thoughts and behaviors center on appearance, with higher scores indicating a greater investment in appearance. These two subscales were translated into Chinese with permission. The factor structure and internal consistency were tested in the control group. The two-factor structure was replicated. ${ }^{19}$ The internal consistency was satisfactory using Cronbach $\alpha$ and was 0.82 and 0.88 for Appearance Evaluation and Appearance Orientation, respectively.

\section{Stigma}

We developed the measurement of stigma, which consists of two subscales. The nine-item Stigma of Deformity measures the negative stereotype of an appearance with significant defects. The Stigma of Deformity consists of items associated with devaluation of ability, decreased popularity, difficulty in making friends of the opposite sex, lacking an opportunity for marriage, being easily insulted, bad fate, self-shame, shame to the family, and feelings of abnormality. Participants were asked to rate the items on a five-point Likert scale, with 1 indicating strong disagreement and 5 indicating strong agreement. Higher scores on the Stigma of Deformity indicated that the person believed more strongly that bad looks would lead to a bad life. The Stigma of Surgery consists of two items that measure the negative impression of using surgery for beauty enhancement. Items are evaluated on a five-point Likert scale, with 1 indicating strong disagreement and 5 indicating strong agreement. Higher scores indicated more difficulty accepting cosmetic surgery as a route for improving physical appearance. Internal consistency using Cronbach $\alpha$ in the control group was 0.88 and 0.71 for Stigma of Deformity and Stigma of Surgery, respectively.

\section{Quality of Life}

The Body Image Quality of Life Inventory quantifies the influence of one's body image experiences on multiple, relevant aspects of psychosocial functioning and well-being in everyday life. ${ }^{16}$ The Body Image Quality of Life Inventory does not measure body image per se; rather, it assesses the impact of one's body image experience on various psychosocial domains of life..$^{20,21}$ Items are evaluated on a seven-point bipolar scale, spanning from +3 , indicating a very positive effect; to 0 , indicating no impact; to -3 , indicating a very negative effect on life. This questionnaire 
was translated into Chinese with permission. Five items (i.e., sexuality, eating, and exercise) were deleted because they were less relevant to the problems of dentofacial deformity, leaving 14 items used in this study. Factor analysis for Body Image Quality of Life was treated as a one-dimensional scale; item loadings on the single-factor solution ranged from 0.53 to 0.86 , with the factor accounting for 53.3 percent of the variance. The internal consistency Cronbach $\alpha$ was 0.93 .

\section{Measures for the Study Group Only: Motivation for Expectation of Surgery}

Motivation of Surgery is a composite score ranging from 0 to 10 , with 0 representing very low willingness and 10 representing very high willingness, to evaluate the degree of willingness to change in six facial areas (i.e., face, nose, cheek, lip, teeth, and chin) before surgery. Higher scores indicate a stronger motivation for surgery. Expectation of Surgery was a multiple choice questionnaire to evaluate a patient's concern regarding surgery (i.e., social interaction, psychological disturbance, and cosmetic improvement).

\section{Procedures}

The study was approved by the National Science Council before data collection. The study group completed the interview and rating scales individually in the outpatient clinic at the craniofacial center 2 weeks before the orthognathic surgery was performed. Before taking part in the measures, all participants signed an informed consent form, after having been fully instructed regarding the study purposes, procedures, and freedom to withdraw.

The control group was recruited from psychology classes. After introduction of the purpose of the scale development, they voluntarily com- pleted these measures, which took approximately 20 minutes.

\section{Statistical Analyses}

To determine the difference between the study and control groups, a series of $t$ tests were used to test the following variables: perception of appearance, body image, stigma, and quality of life, and then two-group discriminate analysis was used to identify the variables in quality of life that best distinguished the study and control groups from one another. Correlation analyses were used to describe the relationships among all variables in the study and control groups. Stepwise multiple regression analysis was used to ascertain the variables that best predicted motivation for surgery.

\section{RESULTS}

\section{Difference between Orthognathic Surgery Patients and Control Groups}

With respect to one's perception of appearance, the orthognathic surgery patients scored lower on their Overall Appearance Rating and Satisfaction of Facial Areas than the control group. With respect to body image, the orthognathic surgery patients scored significantly higher than the control group on Appearance Orientation, but not on Appearance Evaluation. The orthognathic surgery patients experienced significantly more Stigma of Deformity and less Stigma of Surgery than the control group. Concerning body image as it related to quality of life, the orthognathic surgery patients scored significantly lower on the Body Image Quality of Life than the control group (Table 2).

The orthognathic surgery patients scored significant lower on quality of life than the control group in 14 life domains. Two-group discriminate analysis derived one significant discriminate func-

Table 2. Outcome of $t$ Test between Orthognathic Surgery Patients and the Control Group on Appearance Perception, Body Image, Stigma, and Quality of Life

\begin{tabular}{lccc}
\hline & $\begin{array}{c}\text { OGS Patients } \\
(\boldsymbol{n}=\mathbf{7 4})\end{array}$ & $\begin{array}{c}\text { Control Group } \\
(\boldsymbol{n}=\mathbf{1 2 4})\end{array}$ & \multicolumn{1}{c}{$\boldsymbol{t}$} \\
\hline Appearance perception & & $65.78 \pm 14.42$ & $3.240^{*}$ \\
$\quad$ Overall appearance rating & $59.07 \pm 13.57$ & $3.47 \pm 0.54$ & $9.261 \dagger$ \\
$\quad$ Satisfaction of facial areas & $2.75 \pm 0.49$ & $3.07 \pm 0.64$ & 1.114 \\
Body image & $2.96 \pm 0.59$ & $3.58 \pm 0.61$ & $-3.145^{*}$ \\
$\quad$ Appearance evaluation & $3.85 \pm 0.57$ & $2.23 \pm 0.77$ & $2.744^{*}$ \\
Appearance orientation & $1.91 \pm 0.84$ & $2.62 \pm 0.71$ & $-3.468^{*}$ \\
Stigma & $2.98 \pm 0.70$ & $0.79 \pm 0.86$ & $5.555 \dagger$ \\
$\quad$ Stigma of surgery & $0.06 \pm 0.92$ & &
\end{tabular}

OGS, orthognathic surgery.

Independent $t$ test: $* p<0.01 ; \nmid p<0.001$; degree of freedom, 196. 
tion (Wilks' $\lambda=0.79, \chi_{(6)}=45.5, p<0.001$ ) which best distinguished between the orthognathic surgery patients and control groups. Structure matrix, the correlations between discriminating variables and discriminate function, suggested a relative contribution to the discriminate function. As to the orthognathic surgery patients with dentofacial deformities, the impact of quality of life was more important on intrapersonal qualities (i.e., self-worth, confidence, happiness, and satisfaction with life) and social considerations (i.e., work or schooling, meeting new people, and interactions with people) (Table 3).

\section{Correlations of Perception of Appearance, Body Image, Stigma, and Quality of Life}

In comparing the association between subscales of body image and stigma in both groups, significant correlations existed in the control group ( $r=0.28,0.25$ for body image and stigma), but not in the orthognathic surgery patients $(r=$ $0.18,0.05$ for body image and stigma). This im- plied that dentofacial patients sought surgery with the thought that Appearance Evaluation and Appearance Orientation were unrelated, with the same irrelevance as between Stigma of Deformity and Stigma of Surgery. However, those in the control group, who were not preparing for surgery, thought that Appearance Evaluation and Appearance Orientation were positively correlated, with the same positive correlation as between Stigma of Deformity and Stigma of Surgery (Table 4).

For the control group, Appearance Evaluation was significantly related to the Perception of Appearance, Quality of Life, and the Stigma of Deformity $(r=-0.31)$. Appearance Orientation was significantly related to the Stigma of Deformity $(r=0.37)$ and quality of life $(r=$ $0.26)$. For the orthognathic surgery patients, Appearance Evaluation was significantly related to the Perception of Appearance only $(r=0.47$, 0.36 for Overall Appearance Rating and Satisfaction of Facial Areas) and Quality of Life $(r=$ 0.28). Appearance Orientation was significantly

Table 3. Discriminant Analysis between Orthognathic Surgery Patients and Control Group on Body Image Quality of Life

\begin{tabular}{|c|c|c|c|}
\hline & $\begin{array}{l}\text { OGS Patients } \\
(\text { mean } \pm \text { SD) }\end{array}$ & $\begin{array}{c}\text { Control Group } \\
(\text { mean } \pm \text { SD) }\end{array}$ & $\begin{array}{c}\text { Structure } \\
\text { Matrix } \\
\end{array}$ \\
\hline 1. Personal adequacy and self-worth & $-0.16 \pm 1.31$ & $0.98 \pm 1.22$ & 0.844 \\
\hline 6. Experiences at work or at school & $0.09 \pm 0.89$ & $0.78 \pm 0.93$ & 0.697 \\
\hline 5. Experiences when I meet new people & $0.19 \pm 1.12$ & $1.02 \pm 1.16$ & 0.672 \\
\hline 18. Confidence in my everyday life & $-0.19 \pm 1.52$ & $0.84 \pm 1.37$ & 0.670 \\
\hline 19. Happiness in my everyday life & $0.01 \pm 1.26$ & $0.92 \pm 1.29$ & 0.660 \\
\hline 3. Interactions with people of my own sex & $0.27 \pm 1.02$ & $0.99 \pm 1.03$ & 0.653 \\
\hline 4. Interactions with people of the other sex & $-0.08 \pm 1.26$ & $0.81 \pm 1.29$ & 0.653 \\
\hline 10. Satisfaction with $\mathrm{my}$ life in general & $0.08 \pm 1.18$ & $0.85 \pm 1.11$ & 0.635 \\
\hline 7. Relationships with friends & $0.39 \pm 0.87$ & $0.94 \pm 1.01$ & 0.536 \\
\hline 9. Day-to-day emotions & $-0.12 \pm 1.15$ & $0.55 \pm 1.24$ & 0.518 \\
\hline 17. Daily "grooming" activities & $0.26 \pm 1.16$ & $0.79 \pm 1.18$ & 0.425 \\
\hline 2. Masculinity or femininity & $0.31 \pm 1.12$ & $0.78 \pm 1.22$ & 0.371 \\
\hline \multicolumn{4}{|l|}{ 16. Willingness to do things that might call attention } \\
\hline to my appearance & $-0.40 \pm 1.25$ & $0.15 \pm 1.46$ & 0.371 \\
\hline 8. Relationships with family members & $0.24 \pm 0.89$ & $0.60 \pm 0.99$ & 0.352 \\
\hline
\end{tabular}

OGS, orthognathic surgery.

Table 4. Correlations of Appearance Perception, Body Image, Stigma, and Quality of Life in Orthognathic Surgery Patients and the Control Group

\begin{tabular}{|c|c|c|c|c|c|c|c|}
\hline & \multicolumn{2}{|c|}{ Appearance Perception } & \multicolumn{2}{|c|}{ Body Image } & \multicolumn{2}{|c|}{ Stigma } & \multirow[b]{2}{*}{ Quality of Life } \\
\hline & OAppR & SFacA & AEva & AOri & SSur & SDef & \\
\hline OAppR & & $0.50+$ & $0.48+$ & 0.08 & 0.03 & -0.16 & $0.40_{\ddagger}^{+}$ \\
\hline SFacA & $0.30 \dagger$ & & $0.51^{+}$ & 0.08 & -0.16 & $-0.28 \dagger$ & $0.46_{+}^{+}$ \\
\hline AEva & $0.47+$ & $0.36 \uparrow$ & & $0.28 \dagger$ & -0.15 & $-0.31 \dagger$ & $0.53+$ \\
\hline AOri & $0.02^{+}$ & -0.08 & 0.18 & & 0.01 & $0.19 *$ & $0.26 \dagger$ \\
\hline SSur & -0.02 & 0.08 & -0.09 & -0.18 & & $0.25 \dagger$ & -0.05 \\
\hline SDef & -0.02 & -0.21 & -0.07 & $0.37+$ & 0.05 & & -0.09 \\
\hline BIQL & $0.24^{*}$ & $0.23^{*}$ & $0.28^{*}$ & 0.04 & -0.03 & -0.07 & \\
\hline
\end{tabular}

OAppR, Overall Appearance Rating; SFacA, Satisfaction of Facial Areas; AEva, Appearance Evaluation; AOri, Appearance Orientation; SSur, Stigma of Surgery; SDef, Stigma of Deformity; BIQL, Body Image Quality of Life.

Two-tailed correlation: * $p<0.05 ; \uparrow p<0.01 ; \ddagger p<0.001$; OGS patients' correlations appear below the diagonal; the control group's correlations are above the diagonal. 
related to the Stigma of Deformity $(r=0.37)$ only (Table 4).

\section{Predictors of Motivation for Surgery}

Stepwise multiple regression analysis was performed to ascertain which variables would best predict motivation for surgery. Initially, the following variables were entered into the analysis: Overall Appearance Rating, Satisfaction of Facial Areas, Appearance Evaluation, Appearance Orientation, Stigma of Surgery, Stigma of Deformity, and Quality of Life. The results yielded three significant predictors of the regression equation, namely, Satisfaction of Facial Areas $(\Delta R=0.32 ; \beta$ $=-0.523, p<0.001)$, Stigma of Surgery $(\Delta R=$ $0.11 ; \beta=-0.285, p<0.01)$, and Appearance Orientation $(\Delta R=0.06 ; \beta=0.244, p<0.01)$, altogether accounting for 46.2 percent of the total variance $\left(\mathrm{F}_{(3,70)}=21.92, p<0.001\right)$.

\section{Expectation of Surgery}

The basis, with percentages, on which the study group sought surgery was as follows: psychological disturbance (16.2 percent), social interaction problems (5.4 percent), being seen as different (17.6 percent), becoming more beautiful (63.5 percent), and job seeking (4.1 percent). Aesthetic concern seemed to be an explicit and important reason for surgery.

\section{DISCUSSION}

Based on recent research, body image has been the primary motivation for cosmetic treatment. In the current study, perception of appearance reflects the view for specific problems, and was more sensitive in detecting the dissatisfaction component of body image than the satisfaction component. The measures of stigma were uncovered by the negative control for behavioral correctness. These findings of appearance perception and body image suggested that orthognathic surgery patients experienced inferiority in appearance perception but not in explicit body image. Given that the orthognathic surgery patients of this study were shown to be more oriented to their appearance supports theoretical hypotheses. ${ }^{8,11}$

The outcome of stigma supports our hypothesis. As compared with the control group, orthognathic surgery patients were more accepting of surgery as an option for self-enhancement. Orthognathic surgery patients had a higher stigma of deformity, and were especially stigmatized in social situations (i.e., lack of popularity, difficulty in making friends of the opposite sex, decreased op- portunities for marriage, and being easily insulted) but not outwardly in intrapersonal matters (i.e., bad fate, devaluation of ability, self-shame, shame to the family, and feelings of abnormality).

Thompson $^{22}$ proposed that the relation between satisfaction and investment of body image should be orthogonal. In the current study, this notion was confirmed in orthognathic surgery patients, but not in the control group. The different types of associations among variables in the two groups suggested different meanings of appearance investment. Specifically, the control group may be more satisfied with themselves so that they are able to invest more in improving their appearance and destigmatization. In contrast, the orthognathic surgery patients focus their efforts on investing in improvement in appearance, primarily for destigmatization.

Multiple regression analysis identified three significant predictors of motivation of surgery, namely, Satisfaction of Facial Areas, Stigma of Surgery, and Appearance Orientation. It is thus speculated that orthognathic surgery patients may be motivated by dissatisfaction with specific areas, especially the teeth and chins, rather than their overall evaluation. Seeking plastic surgery may be viewed as an investment in appearance improvement. However, it is worthy to note that appearance investment may be somewhat related to Stigma of Deformity, as indicated by their positive correlation coefficient. Therefore, to achieve a substantially satisfactory operation, it is of great importance for the treatment team to fully explain the surgical procedure and potential outcomes before surgery and, meanwhile, to assess and reduce the psychosocial pressure the patients experience in daily life.

\section{CONGILUSIONS}

Patients request orthognathic surgery not only to remedy deformities and functional problems but also to enhance their body image and quality of life and reduce the sense of stigma. Eventually, surgery may help enhance appearance, attractiveness, and self-confidence. In general, such is supported by the results of the present study.

In this study, we took into consideration the existing literature and added a culturally bound factor, stigma, to reveal that the orthognathic surgery patient's appearance orientation may relate to awareness of unattractiveness and sensitivity to social exclusion. Women, in comparison with men, may be more likely to be the targets of gaze or visual inspection..$^{23,24}$ It may thus be culturally acceptable for many women to be more con- 
cerned with and to make greater investment in body image. The findings from the present study that included only female participants need some caution before extrapolation to male subjects. It is yet to be answered whether the stigma of deformity or the stigma of surgery plays any role in male plastic surgery patients in general and male orthognathic surgery patients in particular. These clinically and theoretically relevant questions are worthy of future investigations.

\author{
Sue-Huei Chen, Ph.D. \\ Department of Psychology \\ National Taiwan University \\ No. 1, Sec. 4, Roosevelt Road \\ Taipei 106, Taiwan \\ shchen@ntu.edu.tw
}

\section{ACIKNOWLED GMENT}

The study and data reported in this article were part of a research project granted to Yu-Ray Chen, M.D., by the National Science Council of Taiwan (NSC 93-2314B-182-028).

\section{DISCLOSURE}

None of the authors has a financial interest in any of the products, devices, or drugs mentioned in this article.

\section{REFERENCES}

1. Kiyak, H. A., and Reichmuth, M. Body image issues in dental medicine. In T. F. Cash and T. Pruzinsky (Eds.), Body Image: A Handbook of Theory, Research, and Clinical Practice. New York: Guilford Press, 2002. Pp. 342-350.

2. Kiyak, H. A. Cultural and psychologic influences on treatment demand. Semin. Orthod. 6: 242, 2000.

3. Hunt, O. T., Johnston, C. D., Hepper, P. G., and Burden, D. J. The psychological impact of orthognathic surgery: A systematic review. Am. J. Orthod. Dentofacial Orthop. 120: 490, 2001.

4. Rivera, S. M., Hatch, J. P., and Rugh, J. D. Psychological factors associated with orthodontic and orthognathic surgical treatment. Semin. Orthod. 6: 259, 2000.

5. Lazaridou-Terzoudi, T., Kiyak, H. A., Athanasiou, A. E., and Melsen, B. Long-term assessment of psychologic outcomes of orthognathic surgery. J. Oral Maxillofac. Surg. 61: 545, 2003.

6. Cash, T. F. Body image: Past, present, and future. Body Image 1: $1,2004$.

7. Cash, T. F. Cognitive-behavioral perspectives on body image. In T. F. Cash and T. Pruzinsky (Eds.), Body Image: A Handbook of Theory, Research, and Clinical Practice. New York: Guilford Press, 2002. Pp. 38-46.
8. Sarwer, D. B., Wadden, T. A., Pertschuk, M. J., and Whitaker, L. A. The psychology of cosmetic surgery: A review and reconceptualization. Clin. Psychol. Rev. 18: 1, 1998.

9. Sarwer, D. B., and Crerand, C. E. Body image and cosmetic medical treatments. Body Image 1: 99, 2004.

10. Bolton, M. A., Pruzinsky, T., Cash, T. F., and Persing, J. A. Measuring outcomes in plastic surgery: Body image and quality of life in abdominoplasty patients. Plast. Reconstr. Surg. 112: 619, 2003.

11. Sarwer, D. B., Wadden, T. A., Pertschuk, M. J., and Whitaker, L. A. Body image dissatisfaction and body dysmorphic disorder in 100 cosmetic surgery patients. Plast. Reconstr. Surg. 101: 1644, 1998.

12. Jackson, L. A. Physical attractiveness: A sociocultural perspective. In T. F. Cash and T. Pruzinsky (Eds.), Body Image: A Handbook of Theory, Research, and Clinical Practice. New York: Guilford Press, 2002. Pp. 13-21.

13. Cash, T. F. An investigation of change in body image following cosmetic surgery (Discussion). Plast. Reconstr. Surg. 109: 370, 2002.

14. Henderson-King, D., and Henderson-King, E. Acceptance of cosmetic surgery: Scale development and validation. Body Image 2: 137, 2005.

15. Hatch, J. P., Rugh, J. D., Bays, R. A., et al. Psychological function in orthognathic surgical patients before and after bilateral sagittal split osteotomy with rigid and wire fixation. Am. J. Orthod. Dentofacial Orthop. 115: 536, 1999.

16. Pruzinsky, T., and Cash, T. F. Assessing body image and quality of life in medical settings. In T. F. Cash and T. Pruzinsky (Eds.), Body Image: A Handbook of Theory, Research, and Clinical Practice. New York: Guilford Press, 2002. Pp. 171-179.

17. Cash, T. F. Manual of the Multidimensional Body-Self Relations Questionnaire: The Body Image Quality of Life Inventory. Available from the author at www.body-images.com. 2000.

18. Ching, S., Thoma, A., McCabe, R. E., and Antony, M. M. Measuring outcomes in aesthetic surgery: A comprehensive review of the literature. Plast. Reconstr. Surg. 111: 469, 2003.

19. Brown, T. A., Cash, T. F., and Mikulka, P. J. Attitudinal body-image assessment: Factor analysis of the Body-Self Relations Questionnaire. J. Pers. Assess. 55: 135, 1990.

20. Pruzinsky, T. Enhancing quality of life in medical populations: A vision for body image assessment and rehabilitation as standards of care. Body Image 1: 71, 2004.

21. Cash, T. F., Jakatdar, T. A., and Williams, E. F. The Body Image Quality of Life Inventory: Further validation with college men and women. Body Image 1: 279, 2004.

22. Thompson, J. K. The (mis) measurement of body image: Ten strategies to improve assessment for applied and research purposes. Body Image 1: 7, 2004.

23. Fredrickson, B. L., Roberts, T.-A., Stephanie, M. N., Quinn, D. M., and Twenge, J. M. That swimsuit becomes you: Sex difference in self-objectification, restrained eating, and math performance. J. Pers. Soc. Psychol. 75: 269, 1998.

24. Striegel-Moore, R. H., and Franko, D. L. Body image issues among girls and women. In T. F. Cash and T. Pruzinsky (Eds.), Body Image: A Handbook of Theory, Research, and Clinical Practice. New York: Guilford Press, 2002. Pp. 183-191. 